\title{
Thermal Neutron Computed Tomography at the Argonauta Reactor
}

\author{
Maria Ines Silvani, Gevaldo L. de Almeida, Rosanne C. A. A. Furieri, \\ Instituto de Engenharia Nuclear - CNEN - C.P. 68550, \\ Ilha do Fundão, 21945-970 Rio de Janeiro, RJ, Brazil \\ Ricardo Tadeu Lopes, and Marcelo J. Gonçalves \\ Universidade Federal do RIo de Janeiro - COPPE - Bloco 6. 21945-970, Rio de Janeiro, RJ, Brazil
}

Received on 6 July, 2005

\begin{abstract}
Since 1985 the Argonauta Reactor at the Instituto de Engenharia Nuclear - IEN/CNEN has been used as a source of thermal neutrons to acquire transmission-generated radiographic images. From that time until the end of the last century, some Universities and Research Institutions have as well used the irradiation facilities of this reactor for that purpose and for technical and scientific training of personnel. Quite recently, a joint work program with the Laboratório de Instrumentação Nuclear - LIN/COPPE-UFRJ has been initiated addressing the research in the field of radiographic images using thermal neutrons, with special emphasis on Computer Aided Tomography. Within this frame, different tomographic systems - each one with their intrinsic features have been developed aiming at their use in the industry field which requires the inspection of objects exhibiting different sizes and compositions. This work focuses the developed tomographs presenting their constraints and performances such as Spatial Resolution, Modulation Transfer Function and Density Resolution. The acquired images of some objects and test-bodies are as well presented for the sake of qualitative evaluation. They show a fair agreement with the performance of the tomographic system as expected from the measured quantitative parameters.
\end{abstract}

\section{INTRODUCTION}

A tomographic system is constituted by a source, radiation detectors, ancillary electronics and mechanical devices to move the object under inspection or the source-detector set. Besides these pieces of equipment, special softwares are required to control the movements and to acquire the data and reconstruct the images. Several types of systems have been developed along the last 30 years aiming mainly at a reduction of the scan time, specially for medical applications where the image artifacts caused by certain unavoidable movements such as the patient respiration can be then minimized. The tomographs can be classified according to the their design. The systems of $1^{\text {st }}$ generation employ a narrow radiation beam emitted by a source which moves together with the detector. This set executes a rotation-translation movement, scanning thus the object under inspection. A complete beam translation along the object length furnishes data to obtain a projection of the slice being analyzed. Additional successive projections are gathered by rotating the set source-detector at a certain angle and translating it again, making thus the beam to scan the object under another angle. In the $2^{\text {nd }}$ generation systems the single detector is replaced by a set of 3 to 50 detectors reducing in this way the number of required translations, for a greater amount of data is collected at each translation. In the $3^{\text {rd }}$ generation tomographs the number of detectors is still higher, reaching 200 to 1,000 of them arranged along an arc. Under this configuration the set source-detectors performs only a rotation around the object. The $4^{\text {th }}$ generation contains about 300 to 2,400 detectors disposed along a complete stationary ring. The collimated source emitting a fan-like beam rotates inside and along the circle of detectors. Beyond the medical area, where the object no longer has a well known anatomy, the acquiring time and the image resolution could eventually be non-decisive parameters. Therefore, depending on the specific application, it is necessary to select the more suitable tomograph generation. Furthermore, the employed radiation should be chosen taking into account the size and composition of the object, since the interaction of the radiation with the matter depends upon its nature and energy. The thermal neutron $(\mathrm{E}=0.025 \mathrm{eV})$, is a powerful tool for the non-destructive assay in several fields, thanks to its capability to pass through heavy materials otherwise opaque to X-rays, and due to its strong attenuation for hydrogen. These features allow the detection of hydrogen-bering materials such as drugs and explosives encapsulated within metallic hulls. Other inspections where the neutrons have a stronger potential application than $\mathrm{X}$-rays include metal corrosion, positioning of rubber gasket within metallic components and nuclear reactor fuels. Within the mentioned work program, two tomographic systems have been developed and a third one is under development, all of them using the Argonauta Reactor as source of thermal neutrons. Their features and some acquired images of test-objects are shown in the next sections. The arrangement used at the main channel to collimate and moderate the neutrons coming from the reactor core is shown in Fig.1. The tomographs have been installed at the outlet of this channel where the thermal neutron flux is $4.46 \times 10^{5} \mathrm{n} \cdot \mathrm{cm}^{-2} \cdot \mathrm{s}^{-1}$.

\section{II. $1^{\text {st }}$ GENERATION TOMOGRAPHS}

This system outlined in Fig. 2 was the first one to be developed and uses a $B F_{3}$ neutron detector [1].

This kind of detector is filled with $99 \%$ enriched ${ }^{10} B$ as boron tri-fluoride $\left(B F_{3}\right)$. The reaction ${ }^{10} B(\mathrm{n}, \alpha){ }^{7} \mathrm{Li}$ is responsible for the conversion of the slow neutrons into charged particles. To minimize the neutron scattering, the detector is fully 


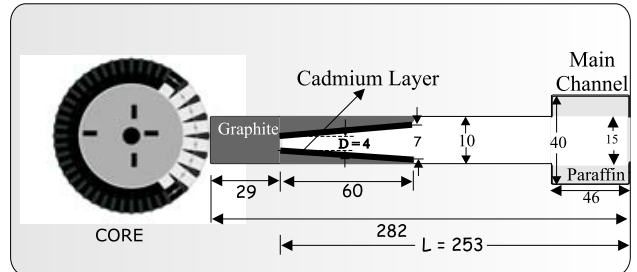

FIG. 1: Collimation and moderation arrangement installed inside the main reactor channel used as source of thermal neutrons for all tomographs.

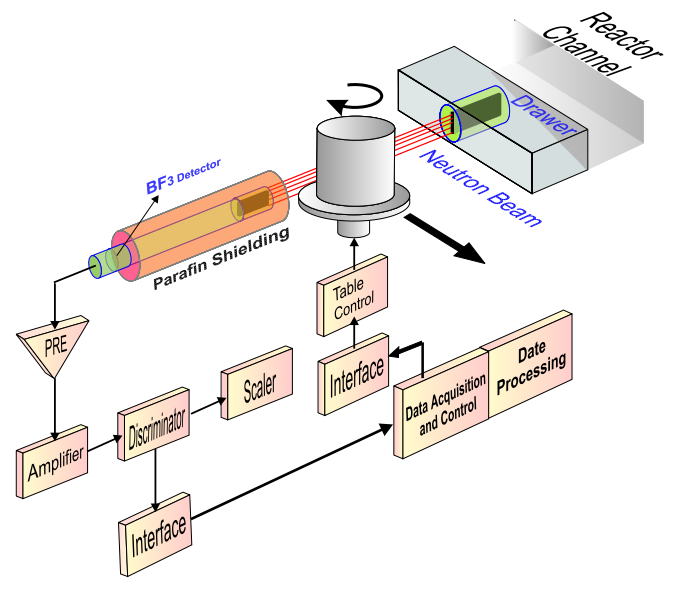

FIG. 2: First Generation Tomographic System.

shielded with a cadmium layer and surrounded by a $20 \mathrm{~cm}$ diameter cylindrical paraffin block, except at its front face. In this system, source and detector are kept static while the object is fixed at the tomographic table which executes rotation and translation movements controlled by a microcomputer. In order to achieve a narrow and parallel beam as required by this type of tomograph, small aperture slit-collimators have been utilized; one close to the source and another at the detector front face, both with the same aperture. To characterize the system and compare the acquired images with those expected from the quantitative parameters, the spatial [2] and density resolutions [3] have been measured by using a homogeneous aluminum cylinder. The tomographies have been carried out with different pair of collimators, with different apertures. The performance of the system has been assessed by using the image of an aluminum cylinder. An average macroscopic cross-section of $0.096 \mathrm{~cm}^{-1}$ has been found which is in good agreement with the literature $\left(\Sigma_{t}=0,099 \mathrm{~cm}^{-1}\right)$ [4]. The Fig.3 shows the impact of the collimator aperture on the spatial and density resolutions, while Fig.4 shows images of a test-object and a small electrical motor acquired with this system.

An aluminum cylinder provided with orifices containing rods of different materials has been used as a test-object. After a scale where the darker gray tonalities are related to the lower macroscopic cross-sections, it can be observed that the system reproduces consistently these parameters. Although tomographs belonging to this generation demand longer acquisition time lapses, they have the capability to handle larger

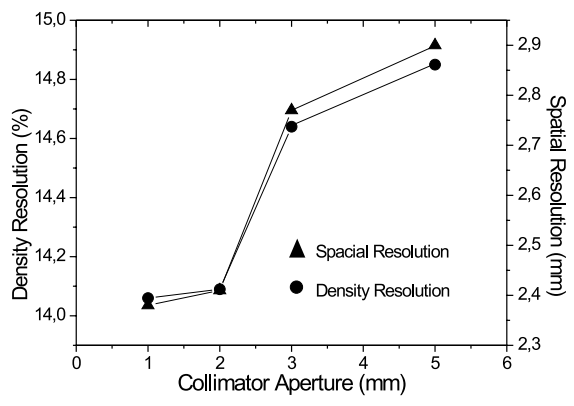

FIG. 3: Density and spatial resolution obtained under several collimator apertures for the neutron tomographic system using a $\mathrm{B} F_{3}$ detector.

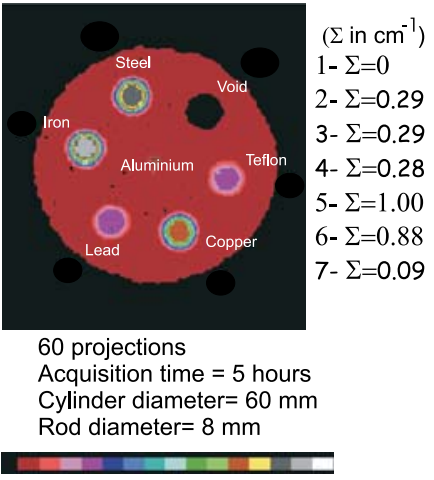

( a)
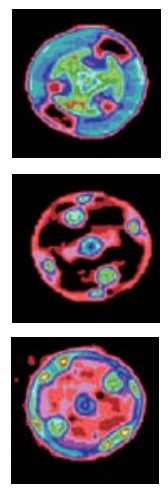

Diameter $=30 \mathrm{~mm}$

(b)
FIG. 4: (a) Test-object. $\Sigma=$ macroscopic cross-section. (b) Crosssections of an electric motor. The tri-pole rotor and the set collectorbrushes are clearly visible.

objects than the detector dimensions, which is an advantageous option for application in the industry.

\section{III. $3^{\text {rd }}$ GENERATION TOMOGRAPHS}

The system outlined in Fig. 5, similar to those ones classified as a $3^{\text {rd }}$ generation equipment, is equipped with a gaseous Position Sensitive Detector-PSD [5,6]. The detector originally designed for X-rays has been modified for use with thermal neutrons. For this purpose, its former carbon window was replaced by a thicker one cast in aluminum, while the original $\mathrm{Ar}-\mathrm{CH}_{4}$ filling-gas has been replaced by ${ }^{3} \mathrm{He}$. Hence, the conversion of the thermal neutrons into charged particles is carried out in the filling-gas itself, which has a cross-section of 5,327 barns for the reaction ${ }^{3} \mathrm{He}(\mathrm{n}, \mathrm{p})^{3} \mathrm{H}$. Due to the high enthalpy $(\mathrm{Q}=764 \mathrm{keV})$, the reaction products are too energetic and cannot be completely absorbed within the detector sensitive region ( $\mathrm{gap}=3 \mathrm{~mm})$. Therefore, to reduce the proton and ${ }^{3} \mathrm{H}$ ranges, improving thus the efficiency, two approaches have been employed. In the first approach propane, and later on an argon-methane mixture have been added to the ${ }^{3} \mathrm{He}$ to enhance 
the stopping power. The filling-gas pressure was kept below $3 \mathrm{~atm}$. In the second approach the gas pressure has been increased to $10 \mathrm{~atm}$. To withstand this higher value, the detector window has been replaced by a thicker one, an engineering constraint which degraded the detector resolution.

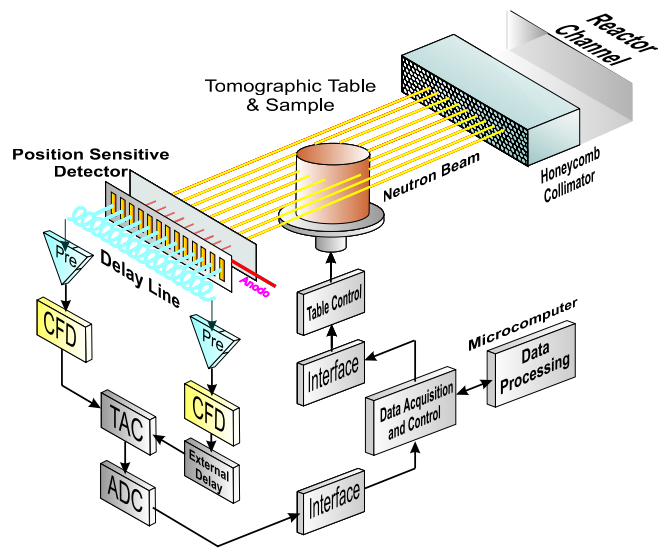

FIG. 5: Third Generation Tomographic System.

The spatial resolution for the system was evaluated through its Modulation Transfer Function-MTF, determined using double-slit collimators with different spatial frequencies, i.e., having different gaps between the slits. This resolution reaches values around $900 \mu \mathrm{m}$. Although systems of this kind limit the maximal size of the object under inspection to the detector window length, it requires a shorter acquiring time lapse than the $1^{\text {st }}$ generation ones, since a sample translation is no longer required. On the other hand, nothing can be done to improve its resolution, for it is tied to the intrinsic resolution of the detector. In the $1^{s t}$ generation systems however, the system resolution can be enhanced by narrowing the collimator apertures. Some images acquired with this system are shown in the figures $6,7,8$ and 9.

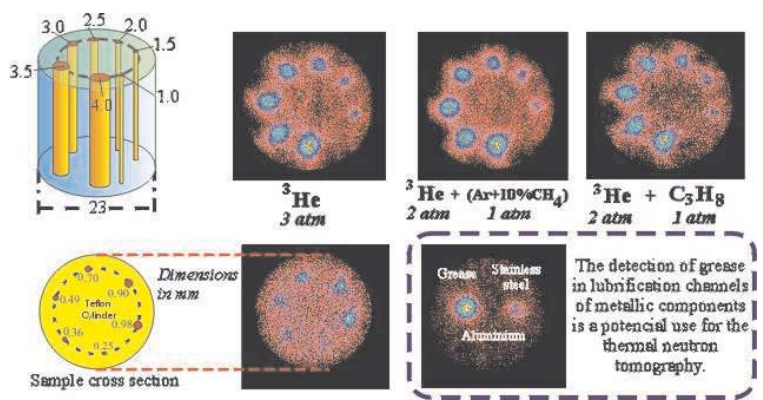

FIG. 6: Images of test-objects acquired with the third generation system. ( 90 projections, acq. time $=90 \mathrm{~min}$ ).

Aluminum and lead are not visible in the teflon matrix for their cross-sections are very close to that of teflon. The brass is readily visible due to its copper contents. The capability of the neutron to pass through heavy metallic materials such as lead and stainless steel allows for instance the detection of explosives encapsulated in metal jackets (used in the air-space industry) and the visualization of its inner structure. This is

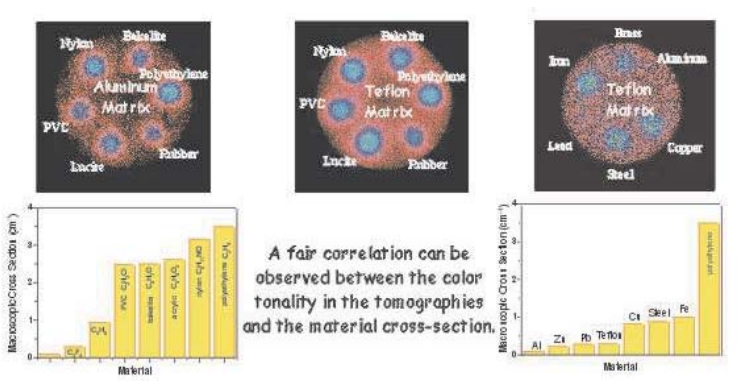

FIG. 7: Images of teflon and aluminum cylinders containing acrylic and metallic inserts.
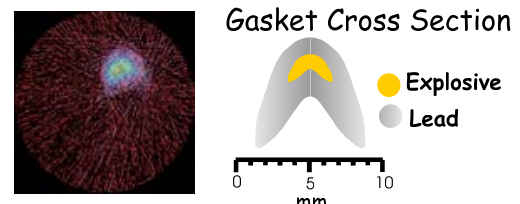

FIG. 8: Image of a lead gasket containing explosive.

important for the non-destructive quality control of the final product where features such as density, voids, alien materials, etc., can be determined.

\section{IV. $5^{\text {th }}$ GENERATION TOMOGRAPHS}

Aiming at the acquisition of 3-D tomographic images in real-time or close to it, a system using a CCD-camera ranked as a $5^{\text {th }}$ generation system, is currently being developed according to the layout presented in Fig. 10.

It is constituted by a scintillation screen - containing materials capable to convert neutrons into charged particles which loose their energy yielding visible light - and an optical system coupled to a CCD camera. As the neutron beam intercepted by the object hits that screen, a light-shadow pattern is drawn, furnishing a neutron attenuation image of the object cross-section. This image is then reflected by the mirror to the optical system placed outside the neutron beam and perpendicular to it, avoiding thus direct neutron radiation damages to the CCD camera. All components are enclosed within an aluminum box lined with a cadmium sheet acting as neutron shielding eliminating thus the scattered neutrons. The CCD camera is coupled to a computer by means of a proper inter-
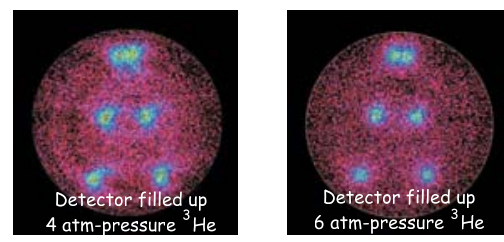

FIG. 9: Images of a $4 \mathrm{~cm}$-diameter aluminum cylinder provided with orifices containing $1 \mathrm{~mm}$-diameter cadmium wires. The clearances between the closest borders of the orifices are 2, 6 and $10 \mathrm{~mm}$ [7]. 


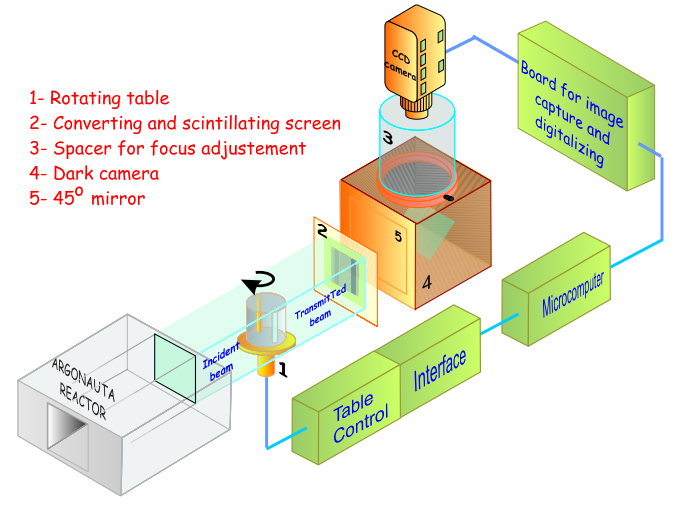

FIG. 10: Tomographic system using a converter-scintillating screen and a CCD-camera.

face and its analogic image is converted to a digital format using a developed software. Currently, this system is being utilized to acquire radiographic images caught by a conventional photographic camera, in order to gather experience and optimize parameters. The Figure 11 shows some qualitative results obtained with this system which is yet under development.
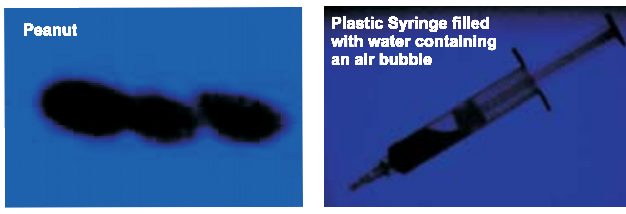

FIG. 11: Thermal neutron radiographic images cast on the converterscintillating screen and caught by a conventional $50 \mathrm{~mm} / \mathrm{f} 1.8$ photographic camera using an ASA 400 film and an exposition time of 30 seconds.
[1] M. I. Silvani, R. T. Lopes, and R. C. A. Furieri, Tomografia Computadorizada 2D com Nêutrons Térmicos e Detector Puntual, V ENAN (2000).

[2] W. A. Kalender, Computer Tomography, 1 ed., MCD Velag, (2000).

[3] P. Reimers, et al, Nuclear Instruments and Methods in Physics Research, 221, 201 (1984)

[4] D. J. Rugles and J. A. Harvey. Neutron Cross Sections, Brookhaven National Laboratory, Upton, New York, (1955).
[5] M. I. Silvani, "Computed Tomography with thermal neutrons and a Position Sensitive Detector," DSc. Thesis, COPPE/UFRJBrazil 2001 (In Portuguese).

[6] M. I. Silvani, et al, Nuclear Instruments and Methods in Physics Research B, 13, 294 (2004).

[7] M. J. Gonçalves, Optimization of a Position Sensitive Detector for use in Tomographic Systems with Thermal Neutrons, M. Sc. Thesis, COPPE/UFRJ-Brazil 2003 (In Portuguese). 